\title{
A Tiered Mobility Management Solution for Cellular/WLAN Integrated Networks with Low Handoff Delay
}

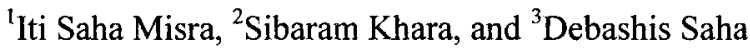 \\ ${ }^{\mathrm{l}}$ Dept. of Electronics and Telecommunication Engg, Jadavpur University, Kolkata, India \\ ${ }^{2}$ Dept. of ECE, College of Engineering and Management, Kolaghat, Midnapur, India \\ and ${ }^{3}$ MIS Group, IIM Calcutta, India, \\ itimisraecal.vsnl.net.in ${ }^{2}$ sianbaerediffmail.com, and \\ ${ }^{3} \mathrm{ds}$ eimcal.ac.in
}

\begin{abstract}
Demand for the Internet services through GPRS/3G networks is proliferating day-by-day. But low bit rate puts constraints on multimedia or mobile business services. In contrast, Wireless Local Area Networks (WLANs) provide high bit rate with low deployment cost. Therefore, integration of these two complementary systems would be the focus of next generation wireless networks. However mobility and handoff management become the key issues for such integration. If a cellular operator deploys as Operator's IP network (OIN), it will be connected to GPRS at Gi interface and to the Internet through firewall. Alternatively, the Internet can be connected directly at $\mathrm{Gi}$ interface and OIN can be connected to the Internet. These connection mechanisms restrict on scalability due to large packet forwarding delay. In this paper, we propose a new way to integrate GPRS/UMTS, OIN and the Internet for better scalability and smaller packet delay. We deploy one gateway for users of cellular (GPRS/UMTS) and WLAN host. The gateway is in OIN and is connected directly with GGSN at Gi interface. The home agent functionalities are distributed in two level hierarchies within the OIN to reduce the packet and handoff delay. The proposed architecture would be useful for large network size.
\end{abstract}

Keywords: 3G/WLAN integration, GPRS, handoff, mobility management, MIP, UMTS, and WLAN.

\section{Introduction}

During last couple of years an increasing number of Internet users, in conjunction with IP based services e.g. e-commerce and applications (e.g., WWW and email), has created a huge demand for wideband access to the Internet. In near future, it is expected that next generation Internet will be the combination of different wireless access technologies, each having different data rates, different coverage areas and different architecture. The interest in cellular/WLAN integration is proliferating for such next generation networks. Design of multimode terminal and mobility management are the key issues of inter-working architectures [4]. The protocol stacks of cellular and WLAN systems can be converged in single dual mode terminal

Please use the following format when citing this chapter:

Misra, I. S., Khara, S., Saha, D., 2007, in IFIP International Federation for Information Processing, Volume 245, Personal Wireless Communications, eds. Simak, B., Bestak, R., Kozowska, E., (Boston: Springer), pp. 206-216. 
equipment $[1,2,3,5,6]$. A software-defined radio (SDR) can dynamically configure itself depending upon best available access network. Tight coupling and loose coupling are two basic methods of cellular/WL,AN integration. Although faster handoff is achieved in tight coupling architecture, it incurs design complexity and congestion in cellular network $[5,7,8]$. Other hand, loose coupling architecture will be more promising for next generation all-IP based heterogeneous network $[9,10]$.

General Packet Radio Services (GPRS) operator can earn huge revenue deploying its own WLAN as operator's IP network. For example, in [1, 2, 3], an operator's IP Network (OIN) is connected at Gi interface with a GPRS network. The Internet is connected to the OIN through firewall. Therefore, the Internet connection for pure GPRS host takes place through OIN. In such an architecture, the hop distance between GPRS host and correspondent node $(\mathrm{CN})$ increases. Obviously, this increases the packet delay compared to the delay when Internet is directly connected to $\mathrm{Gi}$ interface. All traffic travel through core of OIN and may suffer extra delay due to congestion in OIN. The traffic related to cross connection between two OIN would usually be affected by the Internet.

When mobile station (MS) is in GPRS or UMTS network, all incoming packets are routed to GGSN by external IP network. If MS moves to WLAN of OIN, GGSN is the responsible node to forward the packets to IP network. This mandates the deployment of Home Agent (HA) functionality at GGSN so that it can forward IP packet using mobile IP (MIP) based tunneling through OIN. In such case GGSN becomes the unique gateway for GPRS as well as OIN. Therefore, when MS is in WLAN, all incoming packets come via GGSN. GGSN also handles both incoming and outgoing traffic of GPRS service. This architecture increases both packet and handoff delay. To reduce this delay, HA functionality is deployed in OIN. All incoming packets are routed to HA by external IP network. All traffic for GPRS MS is also routed to HA. If HA does not find any MIP binding for destination MS, it may give packets to GGSN. The subscribers having only GPRS connection will also received packets through $\mathrm{HA}$.

We take up the scenario when MS moves within the WLANs under OIN. If MS changes the access router, the MIP based mobility management is always performed with HA. MIP based handoff delay increases with traffic load in ONN.

From the review of related works we find that a better inter-working architecture is very much in demand such that traffic of OIN and Internet do not affect each other. We identify a few more important points in view of user's requirement and operator's interest in cellular/WLAN integration. A cellular user will prefer specific options for WLAN service at hot spots. One, user may prefer for WLAN service with same subscription without changing terminal equipment. Two, user may opt for selective WLAN services of multiple networks. Three, a user with same terminal can have separate subscription for WLAN service of other network.

Again, a cellular operator may wish to have following options. One, it can provide its own WLAN service for its cellular users. Two, the operator may have agreement with other networks so that it can provide their WLAN service to their subscriber.

We address this space and propose an integrated network that fulfills the requirements of users and operators. Moreover, the problem of Mobile IP (MIP) [10] is also taken care of and then we propose tiered mobility agent based solution for fast and seamless handoff intensive environments. 
To validate the proposed architecture, extensive simulation results are provided based on ns-2.26 simulation environment. We emphasize on the design of network model to meet the desired goal for seamless connectivity of large networks with varied mobility patterns of the users and for increasing number of mobile users. Also a performance comparison of the proposed protocol is made with Mobile IP.

\section{Proposed Network Architecture}

Figure 1 shows the inter-working architecture between GPRS, UMTS, Internet and OIN. We deploy a global gateway router for each operator. This router is the gateway for OIN. It is also connected to GPRS or UMTS at Gi interface. This node also provides HA functionality for entire operator's network. We say it a global HA (GHA). GPRS/UMTS operator's IP network and Internet are also connected to it through separate IP level port. GHA provides HA functionality for GPRS MS while moving into IP networks. GHA of GPRS operator and that of UMTS operator can be interconnected for inter-working between GPRS and UMTS operator's IP network. The inter-working networks would be expanded interconnecting other GHA(s) too.

Figure 1 also shows the different roaming scenario for cellular MS. A GPRS MS can move from cellular to own OIN, to Internet or to UMTS ONN. The MS can also move from GPRS OIN to Internet and from there to UMTS OIN. Similarly, UMTS MS can move to its own OIN, to Internet or to GPRS OIN. It can also move from UMTS OIN to Internet and from there to GPRS ONN. Concerned GHA provides HA functionality for mobility management in each of the roaming scenario.

Figure 2 shows the architectural details of OIN. This network provides WLAN service to its cellular users at hot spots. Each OIN has hierarchical structure and connected to cellular network through GHA at Gi interface. We deploy another HA functionality in OIN. These HAs are implemented in lower hierarchy of GHA. We say it as middle HA (MHA). In hierarchical architecture they are deployed in between foreign agent (FA) and GHA. FAs are essentially the access router controlled by MHA. The Operator's WLAN IP network is divided into number of domains. Within a domain, MHA provides the HA functionality. Domains are again divided into subdomains under the control of a FA or AR. An access point (AP) is placed at small WLAN area of each sub-domain under the control of AR. FA and MHA are IPV4 routers with some additional functionality. They can act as proxy $\mathrm{HA}$ for their respective service areas.

Four types of mobility scenario are considered for the proposed cellular/WLAN integrated network as given below.

Case 1: Local mobility - across the APs, MS moves from one WLAN area to other

Case 2: Micro mobility - across the ARs, MS moves from one sub-domain to other

Case 3: Macro Mobility - across the MHAs , MS moves from one domain to other, and

Case 4: Global Mobility - across the GHAs, MS moves from one network to other. 


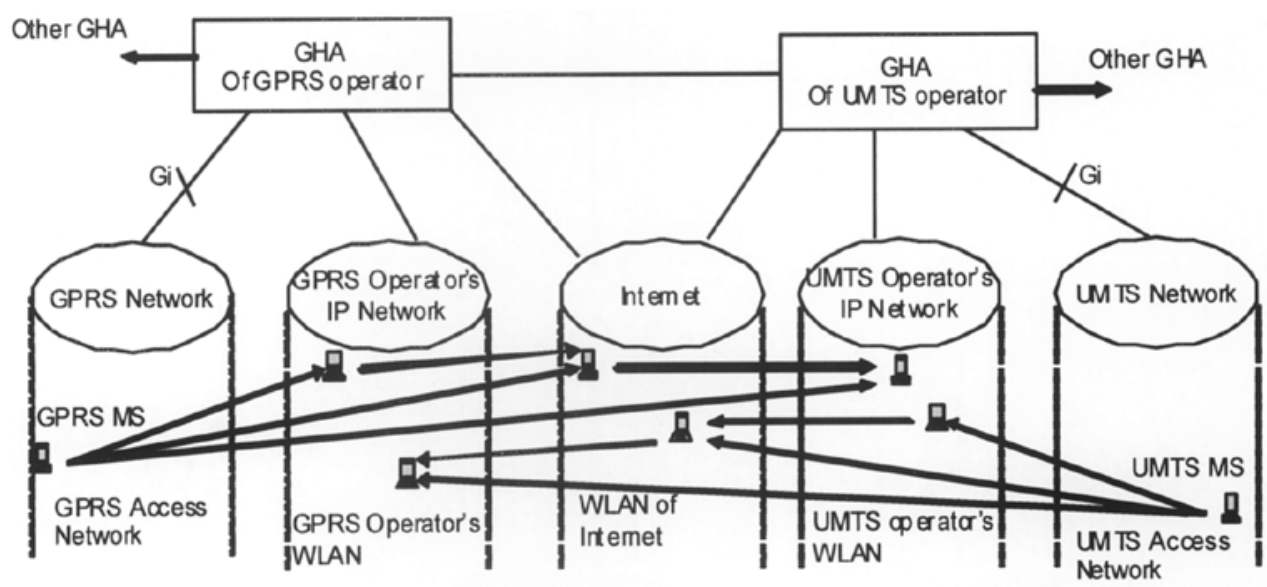

Fig 1: Proposed architecture of œllular (GPRS and UM TS) andWLAN interworking netwo rk.

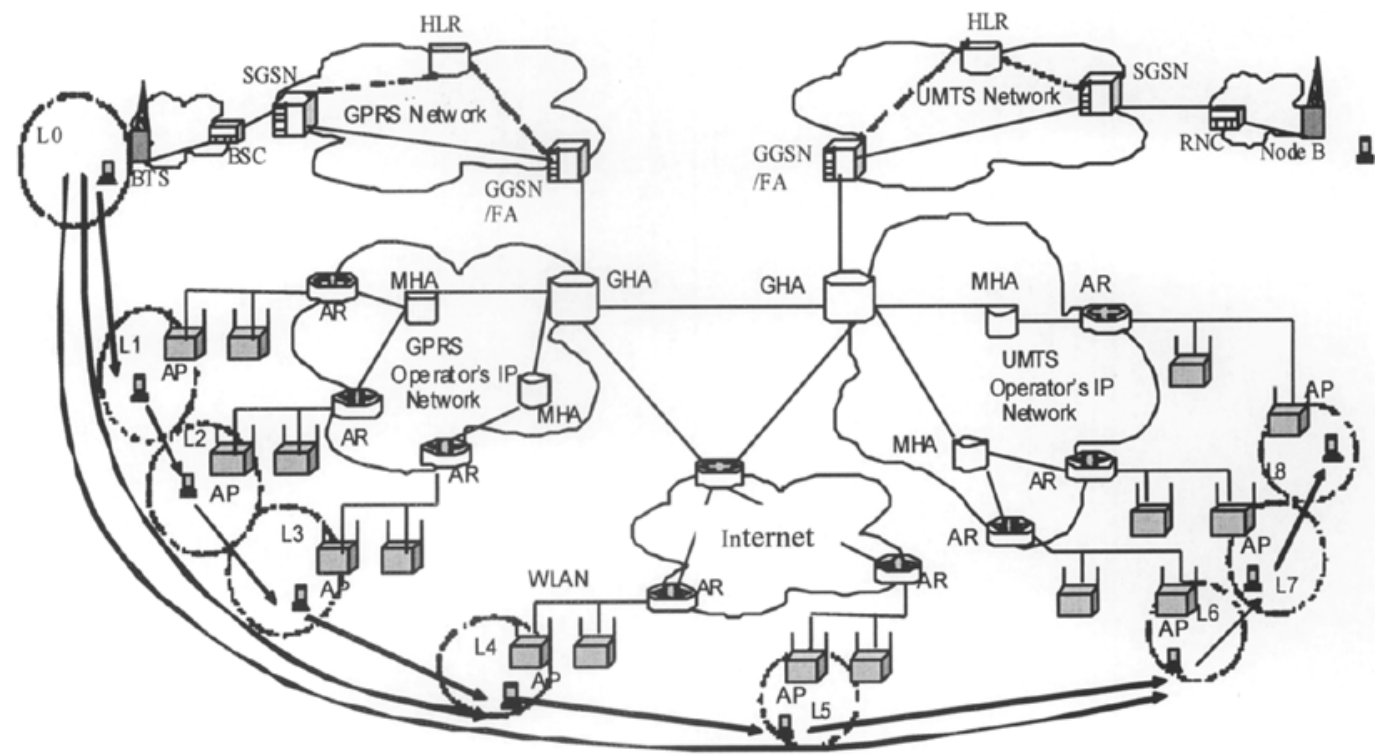

Fig 2 A complete netw $\propto$ k a rchitecture using three level dstribute da gents in qperat a's IP network. 
In global roaming scenario MS performs mobile IP based mobility signaling with $\mathrm{HA}$ at GHA level (example, when MS moves from location L0 to L1). During MIP based handoff signaling, MHAO is updated with the authentication and service profile of the mobile terminal. Subsequently MHAO can provide HA functionality if MS moves within the domain of MHA0. This distributed structure would reduce the registration update latency providing scalability to the network.

\section{Mobility Management of the Proposed Architecture}

The HA functionalities are imposed in GHAs and MHAs. GHA is deployed in gateway router at $\mathrm{Gi}$ interface. MHA can be configured in any $\mathrm{IPv} 4$ router in operator's IP network. Any cellular MS can have additional subscription for WLAN service at hot spots. HA contains the AAA (Authentication, Authorization and Accounting) profiles of WLAN service of cellular MS. GHA acts as gateway router for GPRS/UMTS and operator's IP network. The incoming packet destined for any cellular MS such as GPRS MS is routed to GHA by other network. It checks whether any mobility binding exists for destination MS. If it does not exist GHA assumes that MS might be under cellular coverage. It then sends the packet to GGSN through Gi interface. GGSN handles the IP packet usual way. The mobility entity MHA intercepts each IP packet; both control and data packets. On receipt of MIP registration request packet it checks whether MIP binding already exists in router. If not, it forwards the packet further up in hierarchical network. For example, when first time MS moves from location Lo to location L1, the MHA0 and GHA0 in operator IP network do not have any location information of such MS. Therefore MHA0 cannot provide HA functionality. It forwards the MIP registration request packet to GHA0. GHA0 updates the MIP binding records to map between home address and concerned MHA0. The MIP registration response packet is sent to concern MHA0 providing all AAA information. The MHA0 creates necessary binding information to correlate between home address and Foreign Agent (FA).

Each incoming data packet is intercepted by GHA before it reaches to GGSN. The destination address of each received data is checked by GHA. The destination address of data packet is the home address of the cellular MS. If binding exists for this home address, the GHA retrieves the address of responsible MHA. This data packet is tunneled to MHA using MIP. MHA retrieves the address of responsible FA and tunnel the data packet to it. FA sends the IP packet to destination usual way after decapsulation of the packets.

The implementation of GHA and MHA and their actual position in OIN are the prime task in proposed architecture. The implementation mechanism requires some changes in MIP specifications. The MIP registration request packet remains unchanged. But MIP registration response packet must be modified such that it carries the AAA context. Therefore, a new type of information is to be specified for response packet. The type field can be followed by length and the AAA context. The unused bit pattern can be assigned to designate the new type of information. In a particular hierarchical path, FA must be pre-configured with the address of its MHA. MHA is pre-configured with the address of GHA. FA always forwards the MIP 
registration request packet to its $\mathrm{MHA}$. If MHA cannot provide HA functionality it forwards MIP registration packet to concern GHA. MS has always to send to MIP registration request to $\mathrm{FA}$. MS should acquire the foreign agent care-of address. The MIP registration response packet is to be sent to the agent from which the request was received.

\section{Simulation Results and Discussions}

Creating MIPv4 platform in ns-2.26 simulates the proposed architecture. We develop GPRS tunneling protocol (GTP) to provide the bearer service of GPRS and UMTS for IP packets in cellular network. HA functionality is imposed to provide services needed by GHA and MHA locally. Initially, for MIP based simulation, GHA is replaced by HA and the MHA and AR act as normal IP routers. Then, for the proposed distributed architecture, GHA, MHA and AR are deployed with their respective functionalities. To evaluate the network performance, handoff delay is computed under different roaming scenarios. Typical parameter values used for simulation are provided in Table 1 .

First, handoff delay is computed for the global and macro mobility scenario. In both scenario MS performs handoff signaling with GHA. A global mobility scenario occurs when MS moves from position LO of GPRS to position L1 of OIN as shown in Figure 2. Macro mobility occurs when MS moves from one domain to another domain in OIN. For example such mobility happens when MS moves from position L2 to position L3 in OIN. For such mobility scenarios we compute the handoff delay in pure MIP based system and in GHA/MHA based system. It is seen that the handoff delay in GHA/MHA based system is $8.73 \%$ more than pure MIP based handoff delay. This is because of the user's service; subscription and authentication profiles are imposed in MIP response packet in GHA/MHA based procedure. Thus the size of MIP registration response packet from GHA is more than usual MIP response message. The extra handoff delay is tolerated for first time when global or macro mobility occurs. In such handoff process the MHA gets ready to provide HA functionality for subsequent movement of MS within same sub-domain. Users may frequently perform MIP based handoff signaling due to changing WLAN area. In such cases GHA/MHA based handoff procedure will be more fruitful for a long-term service.

Figure 3 shows the comparison between handoff delays in micro mobility scenario with varying packet size. In MIP based system the HA is implemented at GHA node position. In contrast to micro mobility for GHA/MHA based system; here always-macro mobility management is initiated, whenever MS moves within the IP networks. The handoff delay increases by $32 \mathrm{~ms}$ when packet size is varied from 100 to 1000 bytes as compared to $28 \mathrm{~ms}$ in GHA/MHA based case under same mobility pattern. This effect will be more prominent with the increase of hop distance. Similar results are observed when UMTS MS moves under same environment. In case of a large IP network, the entire IP network domain is divided into sub-domains, which again will be divided into areas. Therefore, the micro and macro mobility scenarios 
may occur frequently. In such situation benefit of handoff delay may be achieved than the usual MIP based technique making the network scalable one.

We compute the handoff delay under varying traffic load in inter-working network. We increase the number of connection between GPRS and Internet hosts.

Table I: Typical values used for simulation

Quque type
Interface Quque length
Link Layer Overhead
Link Layer delay
Adhoc routing
WLAN Host bit rate
GPRS host bit rate
UMTS host bit rate
Trans mitting Power
Physical Channel frequency
TCP Packet size in bytes
BS Omni Antema height
High speed channel between
Two wired node

\author{
DropTail \\ 400 \\ $25 \mu \mathrm{s}$ \\ 50 Hs \\ DSDV \\ $4 \mathrm{Mbps}$ \\ $144 \mathrm{Kbps}$ \\ $2 \mathrm{Mbps}$ \\ 0.5818 w \\ $2.4 \mathrm{Ghz}$ \\ 500 \\ $1.5 \mathrm{~m}$
}

Duplex channel, 40Mbps

We assume that GPRS MSs at location L0 sends traffic to Internet hosts at location L5. All packets pass through GHA to Internet. We compute the handoff delay in micro mobility scenario, when MS moves from location L1 to L2. First we compute the handoff delay in usual MIP based system. Here GHA provides HA functionality. We observe that when GPRS connection is varied from 1 to 10 , the handoff delay increases from $9.44 \%$ to $109 \%$ as shown in Figure 4 . Under same mobility condition and connection environment, we compute the handoff delay in GHA/MHA based system. It is observed that handoff delay remains almost constant. The reason is that the MHA provides the HA functionality. The handoff signals are not dealt by GHA node. The GHA/MHA based handoff signals avoid the queuing and congestion effect of node GHA. The signals also travel shorter hop distance.

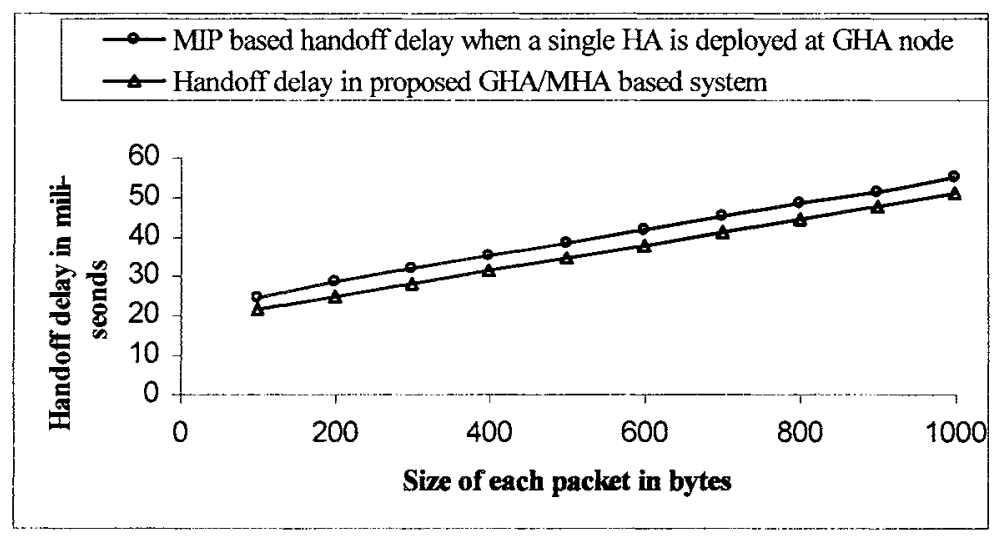

Fig. 3. Handoff vs. Packet size in micro-mobility scenario 
Under same traffic environment the throughput of the system is also computed. We deploy a transmitting MS in location $\mathrm{Ll}$ and corresponding receiving MS at location L3. The receiving MS is employed mobility and enters at location $\mathrm{L} 2$. If receiving MS performs pure MIP based handoff with HA at GHA, then all incoming packets for receiving MS will come via GHA node. But if receiving node performs a GHA/MHA based handoff procedure, then all incoming packets will come via MHA. Because after handoff, MHA behaves as HA for receiving MS and it tunnels all packets to concern FA destined for receiving MS. We compute the throughput at receiving end under varying GPRS connection. It is seen that the throughput reduces by $35 \%$ when number of connections increases by 10 in MIP based system [Fig 5]. In GHA/MHA based system the throughput remains almost constant

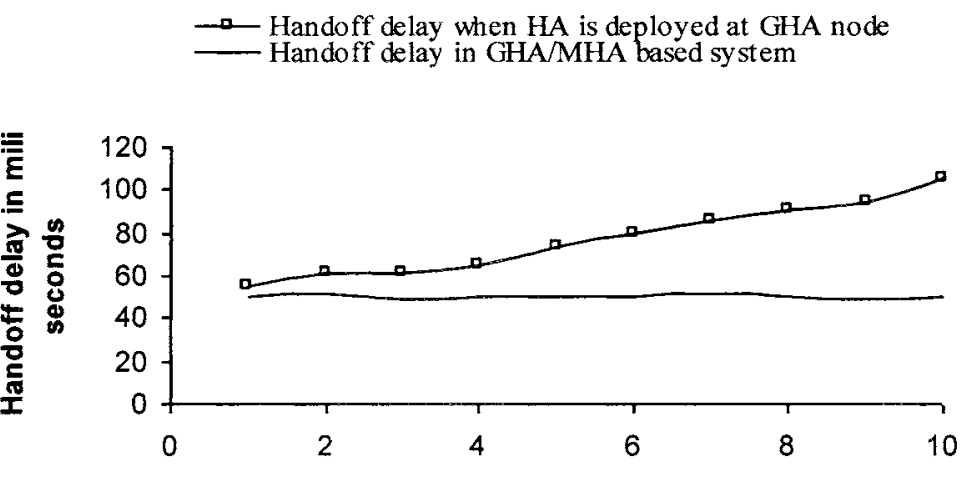

Number of UMTS connections with internet

Fig. 4. Handoff Vs. Number of UMTS connections with Internet

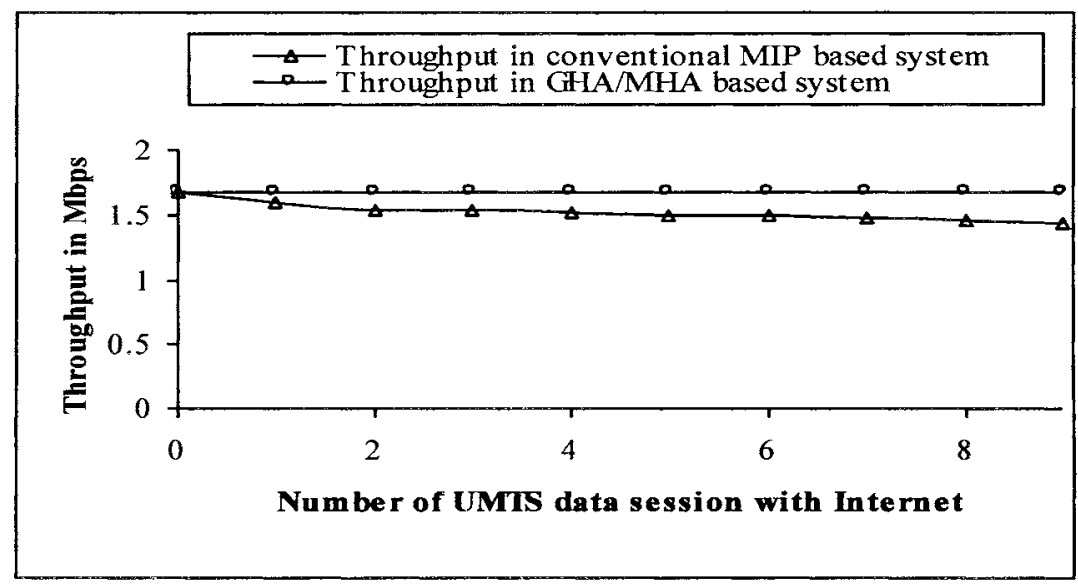

Fig. 5. Throughput vs. number of GPRS connection with Internet in MIP and GMA/HMA based architecture 
The jittering of packets at receiving MS is also observed. The inter-arrival delay of received packets at location L2 for MIP based as well as GHA/MHA based system is computed. It is seen that this delay in pure MIP based system is more fluctuating than that in GHA/MHA based system [Fig 6 and 7]. When receiving MS is in location L2, the incoming packets are affected by the GPRS/Internet traffic at node GHA in MIP based system. This makes uneven packet delay. When receiving MS is in location L2, incoming packets do not suffer the effect of GPRS-Internet traffic.

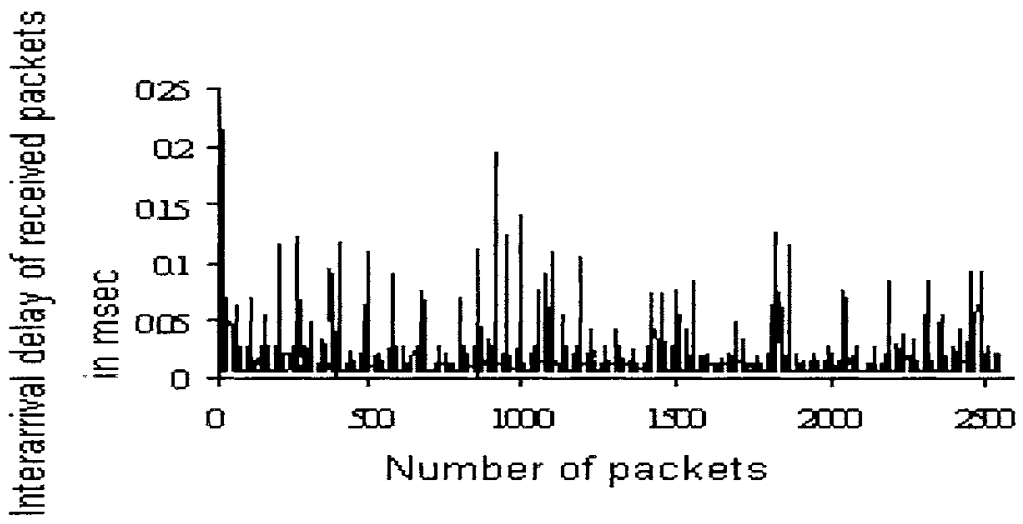

Fig. 6. Inter arrival delay of received packets in MIP based system when transmitting $\mathrm{MS}$ is in $\mathrm{L} 1$ and receiving MS is in L2

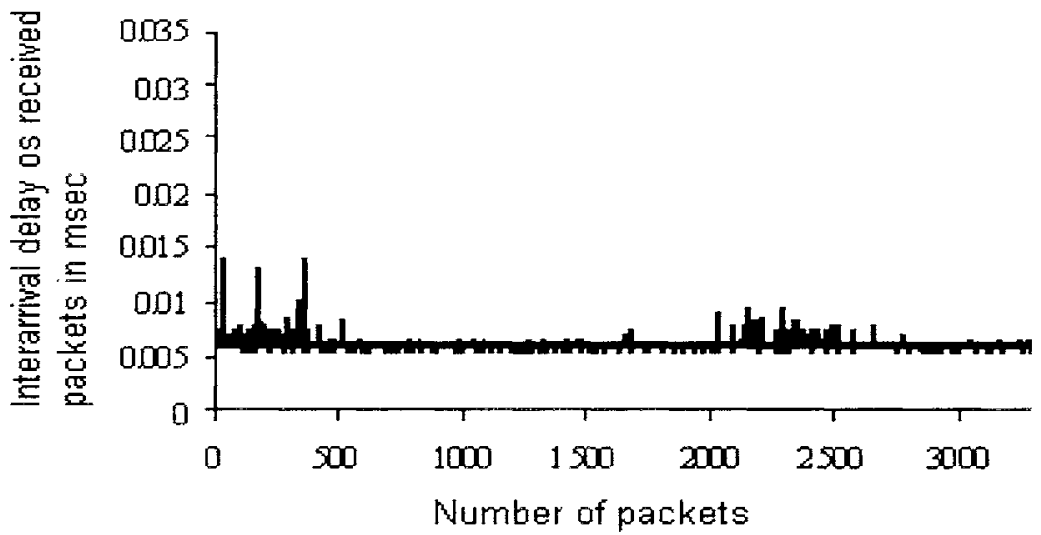

Fig. 7. Inter arrival delay of received packets in GHA/MHA based system when transmitting MS is in $L 1$ and receiving MS is in $L 2$. 


\section{Conclusion}

In proposed cellular/WLAN integrated network, any other operator of GPRS or UMTS technology can join through separate GHA node. Thus architecture allows easy deployment of Operator's IP Networks. Operators can also provide separate corporate networks using other GHA node. In such integrated network, users can have subscription for sole GPRS/UMTS service or for sole WLAN service or for both services. Although as the initial vertical handoff time from cellular to WLAN increases by $5 \%$, for subsequent movements at micro level, the handoff delay reduces substantially. In GHA/MHA based systems, if source and destination exist in the same locality, the traffic does not affect the performance of network outside the locality. Moreover, jitter in GHA/MHA based systems is smoother than that in MIP based systems. Thus the proposed integrated network architecture may be useful for future wireless networks with increased size and traffic.

Acknowledgement: Author Iti Saha Misra is thankful to AICTE for the financial support of this research work under AICTE, CAYT scheme, India.

\section{References:}

1. J. Ala-Laurila, J. Mikkonen, and J. Rinnemaa, "Wireless LAN Access Network Architecture for Mobile Operators", IEEE Communications Magazine, Novmber 2001, pp-82-89.

2. K. Salkintzis, C. Fors and R. Pazhyannur, "WLAN-GPRS Integration for NextGeneration Mobile Data Networks", IEEE Wireless Commun, Oct 2002, pp-112-124.

3. H. Luo, Z. Jiang, B. Kim, N. K. Shankaranarayan and P. Henry, "Integrating Wireless LAN and Cellular Data for the Enterprise" IEEE Comp Soc, March-April 2003, pp-2533.

4. P. Mahonen, J. Rihijarvi, M. Petrova, and Z. Shelby, "Hop-by-Hop toward Future Mobile Broadband IP", IEEE Commun Magazine, Mar 2004, pp. 138-146.J. Clerk Maxwell, A Treatise on Electricity and Magnetism, 3rd ed., vol. 2. Oxford: Clarendon, 1892, pp.68-73.

5. S. Khara, I. S. Mishra and D. Saha, "An Anternative Architecture of WLAN/GPRS Integration" 63rd Vehicular Technology Conference (VTC 05), organized by IEEE , Melbourne, Australia, May 7-10, 0-7803-9392-9/06/\$20.00 (C) 2006 IEEE.

6. H.-W. Lin, J-C. Chen, M-C. Hiang, C-Y. Huang, "Integration of GPRS and Wireless LANs with Multimedia Applications", Proceedings of IEEE Pacific Rim Conference on Multimedia 2002, pp. 704-711.

7. M. Jaseemuddin, "An Architecture for Integrating UMTS and 802.11 WLAN Networks", Proceedings of IEEE Symp on Comp. and Commun.(ISSC-2003), pp. 716723.

8. M. B. R. Murthy and F. A. Phiri, "Performance Analysis of Downward latency in a WLAN/GPRS interworking System." Journal of Computer Science 1(1), Science Publications, 2005, pp 24-27.

9. K. Salkintzis, "Interworking techniques and Architectures for WLAN/3G integration toward 4G Mobile Data Networks" IEEE Wireless Commun, June 2004, pp-50-61. 
216 PWC 2007

10. F. Akyildiz, J. Xie and S. Mohanty "A Survey of Mobility Management in NextGeneration All-IP-Based Wireless Systems", IEEE Wiress Communications, Aug 2004, pp 16-28.

11. M. Shi, X.(S) Shen and J.W. Mark, "IEEE802.11 Roaming and Authentication in Wireless LAN/Cellular Mobile networks." IEEE Wireless Communication, Aug 2004, pp 66-75.

12. C. Perkins, "IP mobility support "IETF RFC 3220 Jan 2002. 